\title{
Effects of pitch distance and likelihood on the perceived duration of deviant auditory events
}

\author{
Elisa Kim • J. Devin McAuley
}

Published online: 27 June 2013

(C) Psychonomic Society, Inc. 2013

\begin{abstract}
When a deviant (oddball) stimulus is presented within a series of otherwise identical (standard) stimuli, the duration of the oddball tends to be overestimated. Two experiments investigated factors affecting systematic distortions in the perceived duration of oddball stimuli. Both experiments used an auditory oddball paradigm where oddball tones varied in both their pitch distance from the pitch of a standard tone and their likelihood of occurrence. Experiment 1 revealed that (1) far-pitch oddballs were perceived to be longer than near-pitch oddballs, (2) effects of pitch distance were greater in low-likelihood conditions, and (3) oddballs in later serial positions were perceived to be longer than oddballs in earlier serial positions. The above effects held regardless of whether oddballs were higher or lower in pitch than the standard. Experiment 2 revealed a pattern of response times in an oddball detection task that generally paralleled the pattern of data observed in Experiment 1; across conditions, there was a negative correlation between detection times and perceived duration. Taken together, the results suggest that the observed effects of oddball pitch, likelihood, and position on perceived duration are at least partly driven by how quickly individuals are able to initiate timing the oddball following its onset. Implications for different theoretical accounts of the oddball effect are discussed.
\end{abstract}

Keywords Temporal processing - Attention: Selective · Music cognition · Sound recognition

E. Kim · J. D. McAuley $(\bowtie)$

Department of Psychology, Michigan State University, East

Lansing, MI 48824, USA

e-mail: dmcauley@msu.edu

\section{Introduction}

As we engage in different activities in our day-to-day lives, the passage of time can seem to ebb and flow in different contexts. Indeed, situations that reduce awareness of the passage of time, such as participating in a particularly engrossing activity, can give the impression that time has sped up (e.g., "time flies when you are having fun"), whereas situations that increase awareness of time, such as boredom and anticipation, can give the impression of time slowing (e.g., "a watched pot never boils"). This article considers factors affecting systematic distortions in perceived event duration that have been associated with an event's predictability.

A number of previous studies have shown that oddball events that deviate from what is expected in an otherwise predictable stream of events tend to be perceived to last longer than expected events with the same objective duration (Pariyadath \& Eagleman, 2007; Seifried \& Ulrich, 2010; Tse, Intrilligator, Rivest, \& Cavanagh, 2004; van Wassenhove, Buonomano, Shimojo, \& Shams, 2008). The general paradigm used in these studies presents participants with a deviant oddball stimulus embedded within a sequence of identical, or otherwise predictable, standard stimuli. The task for each sequence is to judge whether the duration of the oddball stimulus is shorter or longer than the duration of the standard. The basic finding is that individuals tend to overestimate the duration of the oddball stimulus.

Tse et al. (2004) conducted one of the first comprehensive investigations of the oddball effect, manipulating perceptual features of oddball stimuli in a sequence of standard stimuli where the standards ranged in duration from 75 to $2,100 \mathrm{~ms}$. Stimuli mostly consisted of simple visual shapes but also included auditory stimulus conditions. Across stimulus conditions, oddballs were substantially overestimated in duration, with the magnitude of this overestimation ranging, on average, 
from as little as about $25 \%$ for auditory oddballs to as much as nearly $60 \%$ for visual oddballs. The amount of overestimation was found to be influenced by the salience of the oddball stimulus, where salience was operationalized roughly as the amount of perceived change per unit of objective time (e.g., when the oddball was a visually expanding disk, there was more temporal expansion than when the oddball was a stationary disk). The magnitude of the oddball effect also depended on the standard duration, suggesting that some minimal standard duration, estimated to be about $120 \mathrm{~ms}$, was required to obtain the effect.

The theoretical account of the oddball effect proposed by Tse et al. (2004) is that the oddball stimulus receives enhanced attention (similar in form to attentional capture). On this view, enhanced attention to the oddball is hypothesized to increase its perceived duration by increasing the amount of accumulated temporal information over the oddball's extent. From a mechanistic standpoint, this is realized by a pacemaker-accumulator (internal clock) model of timing, which consists of three stages: clock, memory, and decision (Gibbon, 1977; Gibbon, Church, \& Meck, 1984; Treisman, 1963). Of primary interest for the enhanced-attention account is the clock stage, which includes pacemaker, switch, and accumulator components. The pacemaker generates a continuous series of pulses. In response to a to-be-timed stimulus, the switch closes, allowing pulses to flow into the accumulator. The accumulator "collects" the pulses, providing a representation of duration. Attention acts on the internalclock process by increasing the effective number of pulses that flow into the accumulator (Church, 1984; Lejeune, 1998; Meck, 1983); thus, enhanced attention implies more accumulated pulses and longer perceived duration. A related theoretical account proposed by Ulrich, Nitschke, and Rammsayer (2006) is that the oddball effect is not due to enhanced attention per se but, rather, is associated with increased arousal, which is assumed to increase the rate of the pacemaker (Penton-Voak, Edwards, Percival, \& Wearden, 1996; Treisman, Faulkner, Naish, \& Brogan, 1990), also resulting in more accumulated pulses and longer perceived duration.

A different theoretical account is that the oddball effect is an indirect consequence of reduced neural activity in response to the repeated standard stimulus (Pariyadath \& Eagleman, 2007, 2012; Schindel, Rowlands, \& Arnold, 2011). On this repetition suppression view, the temporal expansion of the oddball event is a by-product of reduced neural activity to the repeated or more generally predictable stimulus, rather than the result of enhanced attention to the oddball stimulus. This view assumes that duration is represented by the magnitude of neural response. Thus, because exposure to the repeated standard stimulus produces reduced neural activity, the duration representation for the standard is temporally contracted (i.e., shortened), and an equivalentduration oddball is perceived as longer in comparison. Some support for a repetition suppression account of the oddball effect comes from evidence of a "debut" effect (Pariyadath \& Eagleman, 2007); namely, when participants are presented with a sequence of identical stimuli, the duration of the first stimulus tends to be perceived to be longer than the durations of successive repeated stimuli (see also Rose \& Summers, 1995, for a similar finding). Also supporting a repetition suppression account is the finding that oddballs that occur in later serial positions tend to be overestimated to a greater degree than oddballs that occur in earlier serial positions (Pariyadath \& Eagleman, 2012).

The overarching goal of the present study was to provide new empirical constraints for theory. The experiments reported below are, in part, motivated by the fact that in the general oddball paradigm, the deviant oddball stimulus is, by design, a physically different stimulus (i.e., the oddball is defined by what the stimulus is). The oddball, however, is also necessarily an unlikely stimulus in an otherwise identical series of stimuli. To separate the potential contributions of both types of "oddness" to the oddball effect, we used an auditory oddball paradigm in which one of two types of oddball could occur on each trial. The two types of oddball varied in their physical pitch difference from the standard tone and their likelihood of occurrence.

Participants heard nine-tone sequences consisting of identical (standard) tones and one embedded oddball tone, identified by a different pitch, which occurred in the fifth to the eighth serial position. Oddball tones were either near or far in pitch from the standard, and each oddball pitch was presented on either $75 \%$ or $25 \%$ of the trials (see Fig. 1). To determine whether any effects of oddball pitch distance were absolute or relative effects, we also compared conditions where the near- and far-pitch oddballs were both either higher (see Fig. 1a) or lower (see Fig. 1b) in pitch than the standard. If any pitch effect reflects the relative pitch distance of the oddball from the standard, rather than the absolute pitch height of the oddball, then near- and farpitch oddballs that are both higher versus both lower in pitch than the standard should produce the same pattern of results.

In Experiment 1, participants judged whether a variableduration oddball was shorter or longer than the duration of the standard, while in Experiment 2, participants were asked simply to detect the oddball and respond as quickly as possible for conditions that matched Experiment 1. The motivation for the second experiment was to consider the possibility that the oddball effect might be due, at least in part, to how quickly individuals are able to initiate timing the oddball in response to its onset. Some suggestion that this may be true comes from the study by Pariyadath and Eagleman (2012), which showed that oddballs in later serial positions tend to be overestimated to a greater degree than 
a

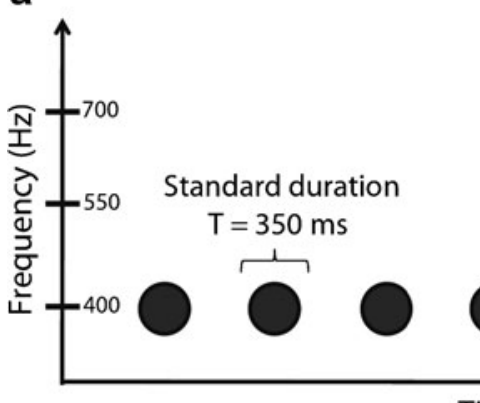

Oddball duration

$\mathrm{T} \pm \Delta \mathrm{t}$
Far oddball

or

Near oddball

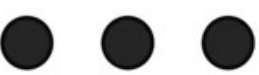

Time (ms)

b

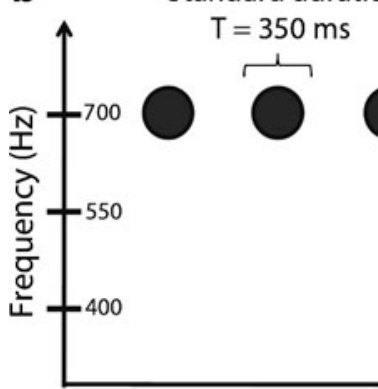

Oddball duration

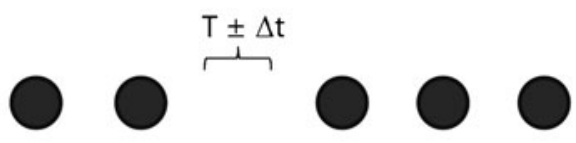

Near oddball

or

Far oddball

Time (ms)

Fig. 1 Diagram illustrating a single trial in the auditory oddball paradigm. Participants heard a sequence of nine tones that included an embedded oddball tone identified by a different pitch that occurred in

oddballs in earlier serial positions. Critically, in this and almost all other oddball studies, the oddball position varied from trial to trial; thus, the later position oddballs afforded greater temporal preparation (i.e., they could be better anticipated). If the speed of detecting the oddball contributed to the degree of lengthening, more generally, then conditions yielding longer perceived oddball durations in Experiment 1 should yield faster oddball detection times in Experiment 2.

\section{Experiment 1}

Method

\section{Participants}

Seventy-two undergraduate students (50 female; 18-24 years, $M=19.1, S D=1.3$ ) from Michigan State University, with self-reported normal hearing, participated in the experiment in return for partial course credit. ${ }^{1}$ Participants varied in number of years of formal music training $(M=3.9, S D=3.8)$.

\footnotetext{
${ }^{1}$ Demographic data from one participant in Experiment 1 and another participant in Experiment 2 was not available due to computer error during data collection.
}

the fifth, sixth, seventh, or eighth position. Oddball tones were presented either higher in pitch (a) or lower in pitch (b) than the standard tones

An additional 14 individuals completed the experiment but were not included in the final sample, due to failure to attend to the task/follow instructions or exceptionally poor performance. ${ }^{2}$

\section{Design}

The design of the experiment was a 2 (Pitch Direction: higher, lower) $\times 2$ (Pitch Distance: far, near $) \times 2$ (PitchLikelihood Pairing: far-75\%/near-25\%, far-25\%/near$75 \%) \times 4$ (Oddball Serial Position: 5th, 6th, 7th, or 8th) $\times$ 9 (Oddball Duration: $-20 \%,-15 \%,-10 \%,-5 \%, 0 \%,+$ $5 \%,+10 \%,+15 \%,+20 \%)$ mixed factorial. Oddball pitch distance, position, and duration were within-subjects variables;

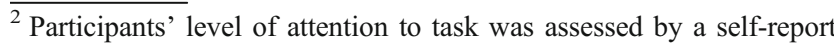
measure of attention and by free response comments collected at the end of the experiment. Participants were asked to rate their attention to the task on a 6-point scale: 1 (little to no attention) to 6 (full attention). Participants were also asked to comment on any aspect of the experiment they had just engaged in. Most participants provided attention ratings of 5 or 6 . Participants who rated their attention as 2 or less or commented that they fell asleep during the experiment ( 1 person for Experiment 1) were excluded from the final sample. Any participants with negative estimates for JND, which can arise if participants do not follow instructions and respond shorter when the oddball is longer than the standard and longer when the oddball is shorter than the standard, were also excluded.
} 
pitch direction and pitch-likelihood pairing were betweensubjects variables. Separate groups of participants completed the oddball-higher $(n=39)$ and oddball-lower $(n=33)$ pitch direction conditions. Within each pitch direction condition, participants were randomly assigned either to the far -75 $\% /$ near-25\% pitch-likelihood pairing condition where the far-pitch oddball occurred on $75 \%$ of trials and the nearpitch oddball occurred on $25 \%$ of trials (oddball-higher, $n=$ 20 ; oddball-lower, $n=18$ ) or to the far- $25 \% /$ near-75\% pitch-likelihood pairing condition where the far-pitch oddball occurred on $25 \%$ of the trials and the near-pitch oddball occurred on $75 \%$ of the trials (oddball-higher, $n=19$; oddball-lower, $n=15$ ).

\section{Stimuli and apparatus}

Standard stimuli were 350-ms sine tones. In the oddballhigher condition, the standard was $400 \mathrm{~Hz}$, and the near- and far-pitch oddballs were $550 \mathrm{~Hz}$ (standard $+150 \mathrm{~Hz}$ ) and $700 \mathrm{~Hz}$ (standard $+300 \mathrm{~Hz}$ ), respectively. In the oddballlower condition, the standard was $700 \mathrm{~Hz}$, and the near- and far-pitch oddballs were $550 \mathrm{~Hz}$ (standard $-150 \mathrm{~Hz}$ ) and $400 \mathrm{~Hz}$ (standard $-300 \mathrm{~Hz}$ ), respectively. The interonset interval between successive stimuli was fixed at $700 \mathrm{~ms}$. Stimuli were generated using MATLAB software (The Mathworks, Inc.) and were presented at a comfortable listening level over Sennheiser HD-280 Pro headphones (Old Lyme, CT). Tone presentation and response collection were controlled by E-Prime 2.0 software (Psychology Software Tools, Inc.) running on a Dell Optiplex 760 series computer with Creative Sound Blaster Audigy soundcard installed. Participants made responses using a serial button response box.

\section{Procedure}

On each trial, participants heard an isochronous sequence of nine tones that consisted of eight 350-ms standard tones and one embedded oddball tone that varied in duration from trial to trial (Fig. 1). Participants judged whether the oddball duration was shorter or longer than the standard duration by pressing one of two labeled buttons on a response box. The variable-duration oddball was presented at either the near pitch or the far pitch in the fifth, sixth, seventh, or eighth sequence position. The duration of the oddball took on one of nine values that varied relative to the standard duration, $T=350 \mathrm{~ms}$; these were $-20 \%,-15 \%,-10 \%,-5 \%, 0 \%$, $+5 \%,+10 \%,+15 \%$, and $+20 \%$, corresponding to durations of $280,297.5,315,332.5,350,367.5,385,402.5$, and $420 \mathrm{~ms}$, respectively. Far-pitch and near-pitch oddballs occurred in different proportions, depending on the assigned pitch-likelihood pairing condition: far- $75 \% /$ near- $-25 \%$ versus far $-25 \% /$ near $-75 \%$.
Experimental blocks were preceded by a 12 -trial practice block consisting of 3 trials presenting the $25 \%$ likely oddball (randomly selected from the possible durations) and 9 trials presenting the $75 \%$ likely oddball (once at each oddball duration). Each experimental block consisted of 144 trials, with 36 presentations of the $25 \%$ likely oddball (4 times per duration) randomly intermixed with 108 presentations of the $75 \%$ likely oddball (12 times per duration). Oddball position varied randomly across trials. Participants in both the oddball-higher and oddball-lower pitch direction conditions completed three experimental blocks, for a total of 432 trials (108 trials with $25 \%$ likely oddball and 324 trials with $75 \%$ likely oddball). Participants were required to take a 1-min break every 36 trials and a 2-min break between each block. At the end of the experimental portion, a questionnaire about participant background demographics and any strategies used during the experiment was administered. The entire experiment took approximately $90 \mathrm{~min}$.

\section{Data analysis}

Proportions of longer responses were determined for each of the nine oddball durations in each condition. Response proportions were then used to generate psychometric curves for each participant in each condition. Resulting curves were used to derive estimates of the point of subjective equality (PSE) and the just noticeable difference (JND) using the ztransform method with response proportions of 0 and 1 replaced by $1 /(2 n)$ and $1-(1 / 2 n)$, respectively, where $n$ is the number of observations (Macmillan \& Creelman, 2005). The PSE corresponds to the duration for which the participant responds longer $50 \%$ of the time. The JND is half the stimulus-duration distance between the 25th and 75th percentiles of the psychometric curve and represents a discrimination threshold estimate. For the JND estimates, we randomly sampled observations in the $75 \%$ likelihood condition so that the number of sampled observations matched the number of observations in the $25 \%$ likelihood condition to eliminate known estimation biases associated with differences in the number of observations across conditions (e.g., Hautus, 1995). JNDs are reported herein as a percentage of standard duration, $T=350 \mathrm{~ms}$. For the oddball-higher pitch direction condition, $R^{2}$ measures of goodness of fit for the estimated psychometric curves ranged between .83 and .93 across conditions. For the oddball-lower pitch direction condition, $R^{2}$ measures of goodness of fit for the estimated psychometric curves ranged between .79 and .93 across conditions. PSEs and JNDs were subjected to mixed-measures ANOVAs with pitch direction and pitch-likelihood pairing as betweensubjects variables and pitch distance and position as withinsubjects variables. 


\section{a}

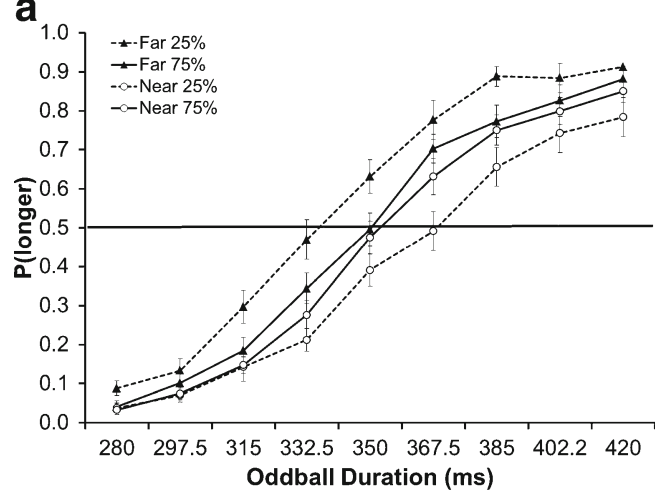

b

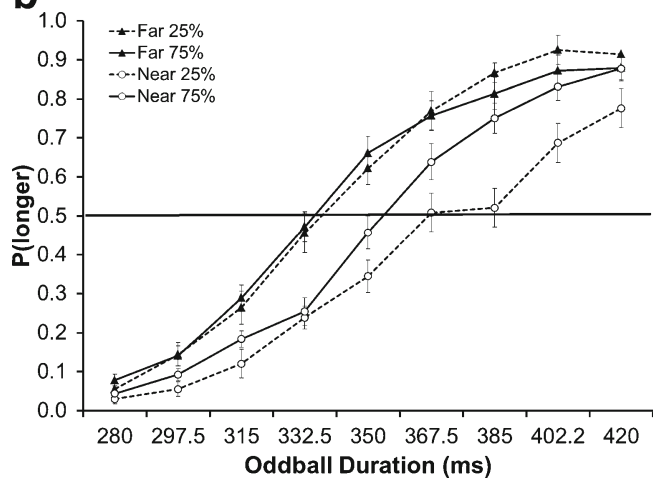

Fig. 2 Mean proportions of longer responses, with standard error bars, as a function of oddball duration for far-pitch oddballs (filled triangles) and near-pitch oddballs (open circles) in $25 \%$ likelihood (dotted lines) and $75 \%$ likelihood (solid lines) conditions for oddballs higher in pitch than the standard (a) and oddballs lower in pitch than the standard (b)

\section{Results}

Figure 2 shows proportions of longer responses as a function of oddball pitch distance and likelihood for the oddball-higher pitch direction condition (Panel a) and the oddball-lower pitch direction condition (Panel b). Corresponding estimates of PSE are shown in Fig. 3a and b, respectively. The 2 (Pitch Direction: higher, lower) $\times 2$ (Pitch Distance: near, far $) \times 2$ (Pitch-Likelihood Pairing: far-75 \%/near-25 \%, far-25$\% /$ near-75 \%) mixed-measures ANOVA on mean PSEs showed a significant main effect of oddball pitch distance, $F(1,68)=26.7, p<.001, \eta_{\mathrm{p}}{ }^{2}=.28$, and main effect of pitchlikelihood pairing, $F(1,68)=7.7, p=.007, \eta_{\mathrm{p}}{ }^{2}=.10$, but no main effect of pitch direction, $F(1,68)=0.1, p=.8, \eta_{\mathrm{p}}{ }^{2}=.001$, and no reliable two-way or three-way interactions (all $p \mathrm{~s}>.2$ ).

In general, psychometric curves for far-pitch oddballs were shifted to the left relative to near-pitch oddballs, and estimates of PSEs were shorter (corresponding to longer perceived durations) for far-pitch oddballs $(M=345.9 \mathrm{~ms}$, $S D=22.5 \mathrm{~ms})$ than for near-pitch oddballs $(M=370.8 \mathrm{~ms}$, $S D=38.1 \mathrm{~ms}), t(71)=5.18, p<.001, d=0.61$. Oddball likelihood also affected PSEs, but the effect depended on oddball pitch. Far-pitch oddballs that occurred $25 \%$ of the time were perceived to be somewhat longer $(M=340.8 \mathrm{~ms}$,
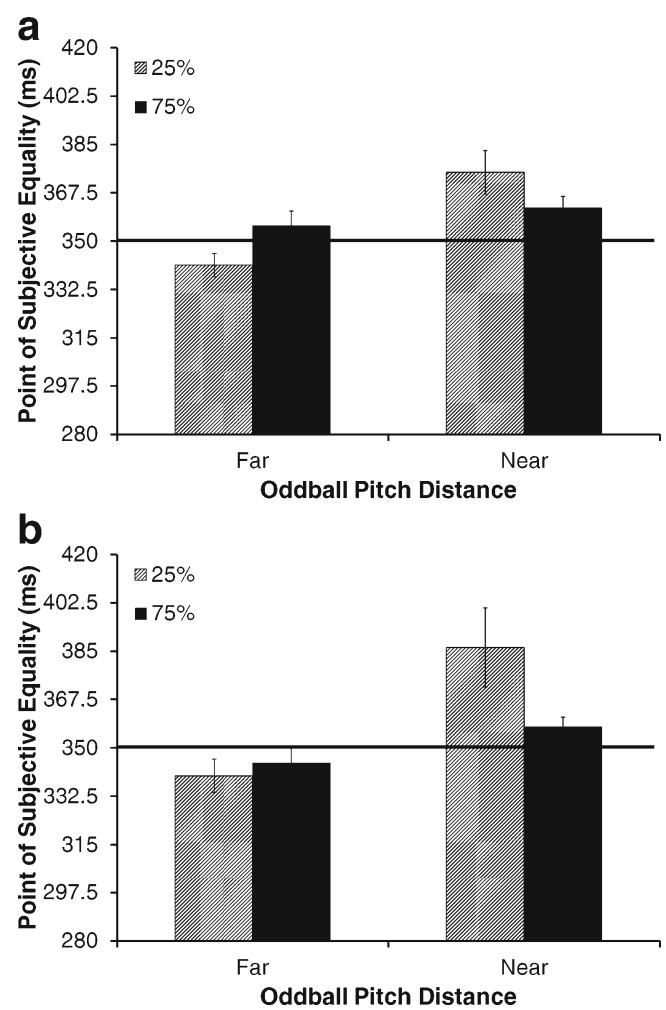

Fig. 3 Mean estimates of point-of-subjective-equality for far-pitch oddballs and near-pitch oddballs in $25 \%$ (shaded bars) and $75 \%$ (solid bars) likelihood conditions for oddballs higher in pitch than the standard (a) and oddballs lower in pitch than the standard (b)

$S D=20.6 \mathrm{~ms})$ than far-pitch oddballs that occurred $75 \%$ of the time $(M=350.5, S D=23.3 \mathrm{~ms}), t(70)=1.87, p=.06, d=$ 0.44. In contrast, near-pitch oddballs that occurred $25 \%$ of the time were perceived to be shorter $(M=380.3 \mathrm{~ms}, S D=$ 48.6) than near-pitch oddballs that occurred $75 \%$ of the time $(M=360.2, S D=16.1), t(70)=2.30, p=.024, d=0.54$.

Table 1 shows mean relative JNDs as a function of oddball pitch distance and likelihood for oddball-higher (left columns) and oddball-lower (right columns) pitch direction conditions. The 2 (Pitch Direction: higher, lower) $\times 2$ (Pitch Distance: near, far) $\times 2$ (Pitch-Likelihood Pairing: far-75\%/ near- $25 \%$, far-25\%/near-75\%) mixed-measures ANOVA on JNDs showed a significant main effect of oddball pitch distance, $F(1,68)=4.98, p=.03, \eta_{\mathrm{p}}^{2}=.07$, but no main effect of pitch-likelihood pairing, $F(1,68)=1.8, p=.18$,

Table 1 Mean just-noticeable-difference as a function of oddball pitch distance and likelihood for Experiment 1 (with standard deviations in parentheses)

\begin{tabular}{lcrr}
\hline Pitch distance & Likelihood & Higher & \multicolumn{1}{l}{ Lower } \\
\hline Near & $25 \%$ & $10.9(4.3)$ & $12.4(7.4)$ \\
& $75 \%$ & $9.6(3.2)$ & $9.8(4.6)$ \\
Far & $25 \%$ & $9.6(3.7)$ & $8.9(2.4)$ \\
& $75 \%$ & $9.8(4.1)$ & $9.8(3.9)$ \\
\hline
\end{tabular}


$\eta_{\mathrm{p}}{ }^{2}=.03$, no main effect of pitch direction, $F(1,68)=0.1$, $p=.8, \eta_{\mathrm{p}}{ }^{2}=.001$, and no reliable two-way or three-way interactions (all $p \mathrm{~s}>.2$ ). Far-pitch oddballs yielded lower discrimination thresholds $(M=9.6 \%, S D=3.6 \%)$ than did near-pitch oddballs $(M=10.7 \%, S D=5.1 \%), t(71)=2.23$, $p=.029, d=0.26$.

Finally, we considered an effect of oddball position on PSEs and JNDs. Table 2 shows mean PSEs and JNDs as a function of position for oddball-higher (left columns) and oddball-lower (right columns) pitch direction conditions. For this analysis, it was necessary to collapse across nearand far-pitch oddballs to obtain enough observations per sequence position to reliably estimate PSEs and JNDs for each subject. A 2 (Pitch Direction: higher, lower) $\times 4$ (Position: 5th, 6th, 7th, 8th) $\times 2$ (Pitch-Likelihood Pairing: far-75\%/near-25\%, far- $25 \% /$ near-75\%) mixed-measures ANOVA on PSEs revealed a significant main effect of position, $F(3,204)=24.03, p<.001, \eta_{\mathrm{p}}{ }^{2}=.26$, but no main effect of pitch-likelihood pairing, $F(1,68)=0.1, p=.75, \eta_{\mathrm{p}}^{2}=$ .002 , no main effect of pitch direction, $F(1,68)=1.3, p=.26$, $\eta_{\mathrm{p}}{ }^{2}=.02$, and no reliable two-way or three-way interactions (all $p \mathrm{~s}>$.6). For the ANOVA on JNDs, there was a main effect of position, $F(3,204)=5.33, p=.003, \eta_{\mathrm{p}}{ }^{2}=.07$, but no main effect of pitch-likelihood pairing, $F(1,68)=0.97$, $p=.33, \eta_{\mathrm{p}}{ }^{2}=.01$, no main effect of pitch direction, $F(1,68)=0.35, p=.56, \eta_{\mathrm{p}}{ }^{2}<.01$, and no reliable two-way or three-way interactions (all $p \mathrm{~s}>.4$ ). Oddballs that occurred in later sequence positions were perceived to be longer (i.e., they had shorter PSEs) than oddballs that occurred in earlier sequence positions. In addition, oddballs that occurred in later positions resulted in better discrimination (smaller JNDs) than did oddballs that occurred in earlier positions.

\section{Discussion}

Results from Experiment 1 showed that far-pitch oddballs were perceived to be longer than near-pitch oddballs, with a larger difference in low-likelihood conditions. Far-pitch oddballs that occurred on $25 \%$ of trials were perceived to be

Table 2 Mean point-of-subjective-equality (PSE) and just-noticeabledifference (JND) as a function of sequence position for Experiment 1 for oddball-higher (left columns) and oddball-lower (right columns) pitch directions (with standard deviations in parentheses)

\begin{tabular}{|c|c|c|c|c|}
\hline \multirow[t]{2}{*}{ Position } & \multicolumn{2}{|l|}{ Higher } & \multicolumn{2}{|l|}{ Lower } \\
\hline & PSE & JND (\%) & PSE & JND (\%) \\
\hline 5 th & $366.0(22.2)$ & $10.1(3.3)$ & $359.2(20.5)$ & $11.0(4.9)$ \\
\hline 6 th & $360.4(24.0)$ & $9.9(3.3)$ & $356.0(21.8)$ & $10.4(5.0)$ \\
\hline 7 th & 353.9 (21.7) & $9.5(3.1)$ & $349.2(16.7)$ & $9.7(3.0)$ \\
\hline 8 th & $352.0(22.0)$ & $9.4(2.7)$ & $347.0(17.5)$ & $9.8(3.5)$ \\
\hline
\end{tabular}

longer than far-pitch oddballs that occurred on $75 \%$ of trials, while near-pitch oddballs that occurred on $25 \%$ of trials were perceived to be shorter than near-pitch oddballs that occurred on $75 \%$ of trials. The same general pattern was observed for oddballs that were higher and lower in pitch than the standard. There was an additional effect of position on PSEs and JNDs; PSEs tended to shorten (perceived durations lengthened) and JNDs tended to be reduced for oddballs in later positions.

It is unlikely that the effect of oddball pitch we observed is an absolute pitch height effect, because we observed the same pattern of results for oddballs that were higher and lower in pitch than the standard. Thus, the results from Experiment 1 suggest that the far versus near difference we observed is an effect of being relatively near or far in pitch from the standard tone, regardless of whether the oddball is higher or lower than the standard. Moreover, it is unlikely that the pitch effect we observed could be due to a difference in the perceived loudness of the oddball, associated with its change in frequency. Note here that the oddball-higher and oddball-lower pitch direction conditions swapped the pitches of the standard tone and the far oddball tone, so if the pitch effect was due to a difference in loudness, we should have not observed the same pattern in the higher and lower pitch direction conditions.

The observed effects of oddball pitch distance (near vs. far) potentially can be explained in terms of either a repetition suppression account or an attention-based account, since both can be interpreted to predict that far-pitch oddballs should produce longer perceived durations than near-pitch oddballs. The observed interaction between pitch and likelihood, however, is harder to interpret from either perspective, since both repetition suppression and attention-based accounts arguably would predict a priori that less likely oddballs would be perceived to be longer than more likely oddballs regardless of pitch, which is not what we observed. Rather, low likelihood enhanced the oddball effect for the far-pitch oddball and reduced the oddball effect for nearpitch oddballs. Moreover, we observed a pronounced shortening of perceived oddball duration in some conditions, rather than the lengthening predicted by repetition suppression or enhanced-attention accounts.

The strongest support for the repetition suppression account is the observed effect of position on PSEs-namely, that PSEs shorten (perceived durations lengthen) for oddballs in later positions. From a repetition suppression perspective, increasing the number of standard stimuli prior to an oddball stimulus should produce greater repetition suppression, thereby increasingly shortening the represented duration of the standard and, consequently, resulting in shorter PSEs for judged oddball durations. One alternative possible explanation of the position effect is that oddballs in later positions are simply more temporally predictable, 
thus enabling faster initiation of a timing process, akin to a variable foreperiod effect on reaction times (RTs; Grondin \& Rammsayer, 2003; Mo \& George, 1977; Niemi \& Näätänen, 1981).

More broadly, this raises the question of whether the pitch effect and the interaction with likelihood may also be related to how quickly individuals are able to initiate timing the oddball. To consider this possibility and the potential role of temporal preparation in the position effect, we conducted a second experiment that used the same design and general procedure as Experiment 1, but rather than having participants judge the duration of the oddball relative to the standard, we had participants simply detect the oddball as quickly as possible. On the basis of the hypothesis that more salient and/or temporally predictable oddballs enable individuals to start timing the oddball sooner, we predicted that RT data from Experiment 2 should parallel the PSE data from Experiment 1, such that conditions that yielded shorter PSEs (longer perceived durations) in Experiment 1 should result in shorter detection times in Experiment 2.

\section{Experiment 2}

\section{Method}

\section{Participants and design}

Forty-eight undergraduate students (32 female; $18-25$ years, $M=19.4, S D=1.6$ ) from Michigan State University, with self-reported normal hearing participated in the experiment in return for partial course credit (see note 1). Participants varied in number of years of formal music training $(M=2.7, S D=$ 4.1). The design parallels Experiment 1; only the task differed. Pitch direction (higher, lower) was crossed with pitch-likelihood pairing (far-75\%/near-25\%, far-25\%/near-75\%). In the oddball-higher condition, approximately half of the participants were assigned either to the far-75\%/near-25\% condition $(n=12)$ or to the far $-25 \% /$ near $-75 \%$ condition $(n=13)$, where both the near- and far-pitch oddballs were higher in pitch than the standard. In the oddball-lower condition, remaining participants were assigned either to the far$75 \% /$ near $-25 \%$ condition $(n=12)$ or to the far $-25 \% /$ near$75 \%$ condition $(n=11)$ where both the near- and far-pitch oddballs were lower in pitch than the standard. Participants' task was simply to listen to each sequence and detect the oddball as quickly as possible by pushing a single button on the response box.

\section{Stimuli and apparatus/materials}

The stimuli, apparatus, and materials were identical to those in Experiment 1.

\section{Procedure}

Participants were instructed to use the index finger of their dominant hand to press the middle button on the response box as soon as they heard the oddball tone amid the ongoing sequence. They received no feedback (i.e., the sequence of tones continued uninterrupted with no beep or other indication of their response being made). At the end of each trial, a fixation cross appeared briefly on the screen, and then the next trial began. All remaining aspects of the procedure were identical to those in Experiment 1. Following the experimental blocks, a questionnaire about participant background demographics and strategies used during the experiment was administered. The entire study took approximately $90 \mathrm{~min}$.

\section{Data analysis}

Data were first trimmed to eliminate any responses that occurred prior to the onset of the oddball (assumed to be accidental/false responses) or that were exceedingly long. ${ }^{3}$ These responses accounted for fewer than $2 \%$ (401 out of $20,633)$ and fewer than $1 \%(20$ out of 20,633$)$ of total responses, respectively. Similar percentages of responses were trimmed in the $75 \%$ and $25 \%$ likelihood conditions for both near- and far-pitch oddballs in the oddball-higher and oddball-lower pitch direction conditions.

\section{Results}

Figure 4 shows mean RTs as a function of oddball pitch and likelihood for the oddball-higher condition (Panel a) and the oddball-lower condition (Panel b). RTs separated by position, pitch distance, and likelihood for the higher and lower conditions are reported in Table 3. The pattern of RTs in Experiment 2 generally paralleled the pattern of PSEs in

\footnotetext{
${ }^{3}$ For most participants, more of the pre-oddball-onset responses were trimmed in the eighth position (277 out of 401), relative to the other three positions. Responses were classified as excessively long and were excluded from analysis if the response occurred after the end of a trial. Seven responses were excluded because they were made more than $62 \mathrm{~s}$ after the trial had ended. These responses indicated that a participant did not respond on the last trial before a 1-min break, causing their buttonpress after the break to be logged as a response. If participants responded after the final tone in the trial sequence, this response was also excluded from analysis, because it was possible on these trials that the participant missed the oddball tone altogether and responded only because they knew the trial was over. For this reason, two responses to trials that presented oddballs in the fifth position, three responses to trials that presented oddballs in the sixth position, seven responses to trials that presented oddballs in the seventh position, and one response to a trial that presented an oddball in the eight position, were excluded from analysis. As indicated in the main text, fewer than $1 \%$ (20 out of $20,633)$ of total responses were excluded for this reason.
} 

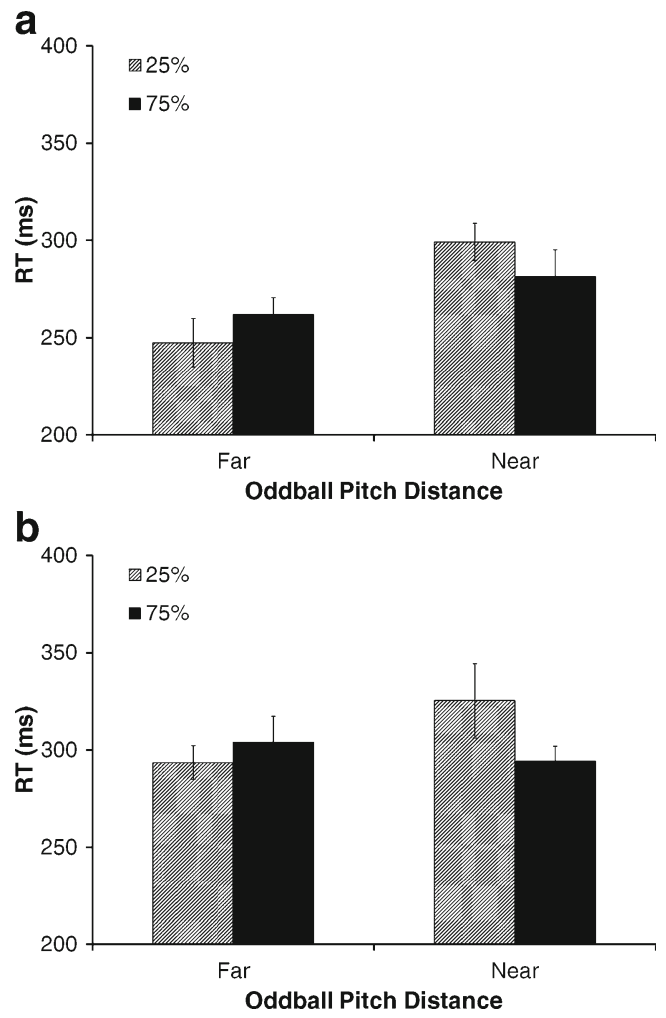

Fig. 4 Mean reaction times (RTs; with standard error bars) from Experiment 2 for far-pitch oddballs and near-pitch oddballs in $25 \%$ (shaded bars) and $75 \%$ (solid bars) likelihood conditions for oddballs higher in pitch than the standard (a) and oddballs lower in pitch than the standard (b)

Experiment 1. The omnibus 2 (Pitch Direction: higher, lower) $\times$ 2 (Pitch Distance: near, far) $\times 4$ (Position: 5th, 6th, 7th, 8th) $\times$ 2 (Pitch-Likelihood Pairing: far-75 \%/near-25\%, far$25 \% /$ near-75 \%) mixed-measures ANOVA on mean RTs revealed a main effect of pitch distance, $F(1,44)=60.92$, $p<.001, \eta_{\mathrm{p}}^{2}=.58$, a main effect of position, $F(3,132)=$ $104.54, p<.001, \eta_{\mathrm{p}}^{2}=.70$, but no main effect of pitchlikelihood pairing, $F(1,44)=2.56, p=0.117, \eta_{p}^{2}=.06$. There was, however, a marginal interaction of pitch distance and pitch-likelihood pairing, $F(1,44)=3.75$, $p=.059, \eta_{\mathrm{p}}^{2}=.08$. There was also a main effect of pitch direction, $F(1,44)=6.82, p=.012, \eta_{\mathrm{p}}{ }^{2}=.13$, an interaction between pitch direction and pitch distance, $F(1,44)=17.05$, $p<.001, \eta_{\mathrm{p}}{ }^{2}=.28$, and a marginal interaction of pitch direction and position, $F(3,132)=2.86, p=.06, \eta_{\mathrm{p}}^{2}=.06$. There was a four-way interaction of pitch distance, position, pitch direction, and pitch-likelihood pairing, $F(3,132)=3.41, p=.037, \eta_{\mathrm{p}}{ }^{2}=$ .07 . There were no other reliable two-way or three-way interactions (all $p \mathrm{~s}>.15$ ).

Paralleling the pattern of results in Experiment 1, RTs were faster for far-pitch oddballs $(M=275.9 \mathrm{~ms}, S D=$ $44.1 \mathrm{~ms})$ than for near-pitch oddballs $(M=299.8 \mathrm{~ms}, S D=$ $48.1 \mathrm{~ms}), t(47)=6.71, p<.001, d=0.97$. Likelihood appeared to enhance the effects of pitch in the same manner as in Experiment 1, but the pattern was less reliable. As was predicted, far-pitch oddballs that were $25 \%$ likely were responded to faster than far-pitch oddballs that were $75 \%$ likely (far- $25 \%, M=268.7 \mathrm{~ms}, S D=44.2 \mathrm{~ms}$; far- $-75 \%, M=$ $283.1 \mathrm{~ms}, S D=43.7 \mathrm{~ms}$ ), but not significantly so, $t(46)=1.14$, $p=.13, d=0.33$, one-tailed. Also as was predicted, near-pitch oddballs that were $25 \%$ likely were responded to slower than near-pitch oddballs that were $75 \%$ likely (near- $25 \%, M=$ $312.3 \mathrm{~ms}, S D=53.1$; near-75\%, $M=287.4 \mathrm{~ms}, S D=40.0$ ), $t(46)=1.83, p=.04, d=0.53$, one-tailed. In contrast to the pattern of results in Experiment 1, RTs were faster in the oddball-higher $(M=271.3 \mathrm{~ms}, S D=40.6 \mathrm{~ms})$ than in the oddball-lower $(M=303.8 \mathrm{~ms}, S D=44.0 \mathrm{~ms})$ pitch direction condition, $t(46)=2.66, p=.011, d=0.80$, two-tailed. Paralleling the position effect for PSEs observed in Experiment 1, oddballs that were presented in later sequence positions were responded to more quickly than oddballs presented in earlier sequence positions.

Finally, we examined the extent to which oddball detection times (RTs) in Experiment 2 predicted perceived duration (PSEs) in Experiment 1 for each of the 32 combinations of oddball pitch direction (higher, lower), pitch distance (near, far), likelihood ( $25 \%$ and $75 \%$ ), and position (5th, 6th, 7th, or 8th). Results, shown in Fig. 5, reveal a significant positive relationship between RTs and PSEs, $r(30)=.55, p=.001$.

Table 3 Mean reaction times as a function of oddball sequence position, oddball pitch distance, and likelihood from Experiment 2 for oddballhigher (left columns) and oddball-lower (right columns) pitch directions (with standard deviations in parentheses)

\begin{tabular}{|c|c|c|c|c|c|c|c|c|}
\hline \multirow[t]{3}{*}{ Position } & \multicolumn{4}{|l|}{ Higher } & \multicolumn{4}{|l|}{ Lower } \\
\hline & \multicolumn{2}{|l|}{ Near } & \multicolumn{2}{|l|}{ Far } & \multicolumn{2}{|l|}{ Near } & \multicolumn{2}{|l|}{ Far } \\
\hline & $25 \%$ & $75 \%$ & $25 \%$ & $75 \%$ & $25 \%$ & $75 \%$ & $25 \%$ & $75 \%$ \\
\hline 5 th & $327.8(36.0)$ & $333.6(91.1)$ & $283.0(53.6)$ & $298.5(38.1)$ & $390.1(112.6)$ & $337.4(24.3)$ & $347.9(51.4)$ & $363.3(72.9)$ \\
\hline 6 th & $303.9(39.0)$ & $287.5(45.9)$ & $257.1(55.1)$ & $266.1(33.4)$ & $319.0(57.6)$ & $297.6(24.9)$ & $307.6(37.0)$ & $311.8(47.4)$ \\
\hline 7 th & $290.3(37.1)$ & $265.8(37.3)$ & $239.0(43.9)$ & $253.1(32.0)$ & $308.1(58.5)$ & $288.7(32.0)$ & $275.7(32.0)$ & $287.5(42.0)$ \\
\hline 8 th & $275.1(32.8)$ & $232.7(42.5)$ & $204.4(54.7)$ & $229.5(23.9)$ & $280.4(57.2)$ & $249.8(47.3)$ & $237.7(46.2)$ & $251.5(31.5)$ \\
\hline
\end{tabular}




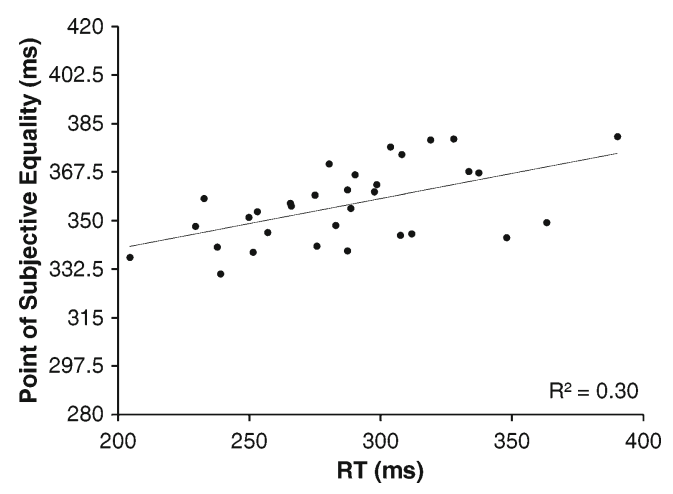

Fig. 5 Scatterplot showing the relationship between reaction times (RTs) in Experiment 2 and mean estimates of point-of-subjective-equality in Experiment 1 for the 32 combinations of oddball position (5th, 6th, 7th, 8th), pitch distance (near, far), likelihood (25\%, $75 \%)$, and pitch direction (higher, lower)

\section{Discussion}

Response times in the oddball detection task varied as a function of oddball pitch distance, likelihood, and position in a manner that generally paralleled the pattern of PSEs in Experiment 1 . Consistent with the possibility that the effect of position on PSEs observed in Experiment 1 reflects a form of temporal preparation akin to a variable foreperiod effect, oddballs that occurred in later serial positions were responded to more quickly than oddballs that occurred in earlier serial positions. Moreover, far-pitch oddballs were detected faster than near-pitch oddballs, with a greater effect of pitch when the oddballs were rare, but less reliably so than in Experiment 1. One reason for the somewhat weaker effects for oddball detection times in Experiment 2 may be due to insufficient power. Experiment 2 also differed from Experiment 1 in that, in Experiment 2, we observed an effect of pitch direction on RTs, which was not observed for PSEs in Experiment 1. The reason for this difference is, at present, not clear. Nonetheless, the results of the two experiments taken together provide initial support for the view that systematic distortions in the perceived duration of auditory oddballs may be at least partly determined by differences in how quickly individuals are able to initiate timing the oddball following its onset.

\section{General discussion}

Five main findings emerged from the present investigation. First, in Experiment 1, far-pitch oddballs were perceived to be longer than near-pitch oddballs, regardless of whether the near- and far-pitch oddballs were higher in pitch or lower in pitch than the standard. Observing the same pitch distance effect regardless of pitch direction is important because it argues against the possibility that the pitch effect we observed is either an effect of absolute pitch height or associated with differences in perceived loudness. Second, likelihood was found to enhance the pitch effect whereby farpitch oddballs that occurred $25 \%$ of the time were perceived to be longer than far-pitch oddballs that occurred $75 \%$ of the time, while the reverse was true for near-pitch oddballs. Third, oddballs that occurred in later sequence positions were perceived to be longer than oddballs that occurred in earlier sequence positions. Fourth, the pattern of RTs observed for the oddball detection task in Experiment 2 generally paralleled the pattern of PSEs observed in Experiment 1; moreover, how quickly participants were able to detect the oddball in Experiment 2 predicted PSEs obtained in Experiment 1. Finally, discrimination thresholds were slightly lower for farpitch than for near-pitch oddballs and lower for oddballs in later serial positions than in earlier serial positions. In the remainder of the discussion, we consider the implication of the present findings for different theoretical accounts.

Tse et al. (2004) proposed that deviant "oddball" stimuli receive enhanced attention and, thereby, should be perceived to be longer in duration than they would otherwise, on the basis of the assumption that the amount of attention increases the effective accumulation of temporal information. In the context of the present experiments, the enhanced-attention account proposed by Tse et al. would explain the pitch effect we observed but would have difficulty accounting for the mediating effect of likelihood. Overall, the enhancedattention account can be interpreted to predict that the more unexpected an oddball stimulus is, the more attention it should attract. Thus, $25 \%$ likely oddballs should be perceived to be longer than $75 \%$ likely oddballs, which is consistent with our finding for far-pitch oddballs. However, inconsistent with the enhanced-attention account, the reverse was observed for near-pitch oddballs, which were perceived to last longer when presented on $75 \%$ of the trials than when presented on $25 \%$ of the trials.

Overall, these findings can be at least partly accounted for by the repetition suppression explanation of the oddball effect (Pariyadath \& Eagleman, 2007, 2012; Schindel et al., 2011). On this view, the temporal expansion of the deviant oddball event is a by-product of reduced neural activity to the repeated or more generally predictable standard stimulus. Thus, the oddball effect is the result of perceived shortening of the standard duration, rather than perceived lengthening of the oddball. Both the position effect and pitch effect are consistent with this view. Repetition suppression accounts for the position effect because more repetitions of the standard occur for oddballs that are in later sequence positions. Here, we also observed a reliable effect of position on temporal discrimination thresholds, but see Matthews (2011) for a null effect of repetition on temporal discrimination thresholds.

Repetition suppression can account for the pitch effect because, at least in some neural models of repetition 
suppression, the magnitude of the oddball effect would also be expected to scale with degree of physical stimulus difference: The more dissimilar the oddball is in pitch from the standard, the greater the neural activity in response to the oddball (see Grill-Spector, Henson, \& Martin, 2006, for a general review of repetition suppression). Like the enhanced-attention account, however, the repetition suppression account would have some difficulty accounting for the mediating effect of likelihood. In general, the rarer ( $25 \%$ likely) oddball should have been perceived to be longer than the more frequent (75\% likely) oddball, regardless of pitch, which is not what we observed.

Some potential insight about the opposite effects of likelihood in near and far pitch conditions may be gleaned from the work of Jones and colleagues on dynamic attending theory and the development of expectations about pitch and time structure in auditory sequences (Jones, 1976; Jones, Moynihan, MacKenzie, \& Puente, 2002; Large \& Jones, 1999; McAuley \& Jones, 2003). Most relevant for this present investigation is Jones, Johnston, and Puente (2006), who showed that the pitch and time structure of a context tone sequence produces expectations in the listener that guide their attentional focus for upcoming target tones. Specifically, pitch discrimination of a target tone was found to be better when the target tone (1) was presented within the expected pitch range of the auditory context and (2) occurred at an expected time point based on an extrapolation of the context rhythm. Applied to the present finding of a pitchdependent effect of likelihood (in the $25 \%$ condition, pitch effects were greater), one possibility is that successive trials establish the context for the development of pitch expectancies, such that when an oddball occurs with a $75 \%$ probability, the pitch of that oddball, together with the repeated standard tone, establishes an expected oddball pitch. Thus, when the near-pitch oddball occurs on $75 \%$ of the trials, the unlikely far-pitch oddball is a more deviant oddball and, thus, may be more detectable. Conversely, when the farpitch oddball occurs on $75 \%$ of trials, the unlikely nearpitch oddball is a less deviant oddball and, thus, may be less detectable. RT data bear out this asymmetric pattern. This perspective refines the concept of oddball saliency to mean the degree to which a to-be-judged stimulus fits/misfits with the pitch expectancy for an oddball, which is dynamically determined by ongoing context.

Although the finding of longer perceived durations for later position oddballs provides support for a repetition suppression account, the present study has provided some initial evidence that the effect of position could also be due to the increase in temporal preparation that is possible for later position oddballs. As the elements of the sequence unfold, participants can be more and more certain that an oddball will occur; that is, they can develop a temporal expectation for the occurrence of the oddball stimulus. Shorter observed
RTs in the detection task (Experiment 2) for later position oddballs are consistent with this interpretation, which are also reminiscent of a variable foreperiod effect on RTs (Klemmer, 1956; Niemi \& Näätänen, 1981).

Some converging support for a temporal preparation interpretation of the position effect comes from several studies (Grondin \& Rammsayer, 2003; Mo \& George, 1977). Grondin and Rammsayer found longer perceived duration of intervals presented after longer foreperiods when the duration of the foreperiod interval was variable from trial to trial. This is analogous in the present investigation to having the sequential position of the oddball stimulus randomly vary from trial to trial. Oddballs in later sequential positions have a longer foreperiod than do oddballs in earlier sequential positions. Mo and George also found that foreperiod influenced temporal reproductions and RTs to a visual stimulus equivalently. Specifically, longer foreperiods (when the length of the foreperiod was unpredictable) resulted in both longer temporal reproductions and shorter RTs.

More broadly, the consistent relationship between the RTs in Experiment 2 and PSEs in Experiment 1 as a function of oddball pitch distance, likelihood, and position suggests a common explanatory source of the observed temporal distortions - namely, in how quickly individuals are able to initiate (start) timing in response to the oddball onset. A number of predictions follow from this hypothesis. Generally speaking, conditions that influence RTs in the detection of an event (as in the foreperiod literature discussed above) should also affect perceived duration in a similar manner. For example, previous studies examining effects of foreperiods on RTs have shown that increasing either occurrence uncertainty or temporal uncertainty of a target stimulus tends to produce longer RTs for detecting the target (Drazin, 1961; Gordon, 1967; Klemmer, 1956; see Niemi \& Näätänen, 1981, for a review of foreperiod effects on RTs). Considered in the context of a temporal preparation account of the effect of position on perceived duration reported here and by Pariyadath and Eagleman (2012), including "catch" trials on which no oddball occurs on some proportion of trials should (1) produce longer PSEs (an overall shortening of perceived durations) and (2) reduce, or even eliminate, the effect of position.

One final issue that deserves some comment concerns the large discrepancies observed in the overall magnitude of the oddball effect across studies. The magnitude of the oddball effect we observed in Experiment 1 (i.e., $5 \%$ overestimation to $7 \%$ underestimation) was much smaller than in previous studies and in some cases (e.g., near-pitch oddballs), we observed shortening rather than lengthening. Notably, Tse et al. (2004) found that overestimation of the oddball duration was as large as $60 \%$ but also centered the oddball duration in the direction of oddball overestimation (i.e., 
participants judged durations that were close to the PSE). Most studies, however, have not revealed the same magnitude of distortions in perceived duration as that observed in Tse et al. Pariyadath and Eagleman (2007) found oddball overestimation of only about $12 \%$ in a visual oddball paradigm. In a cross-modal oddball paradigm with temporally predictable oddballs (i.e., oddballs presented in the same position every trial), van Wassenhove et al. (2008) found oddball overestimation of roughly $8 \%$ for expanding disk and increasing pitch oddballs. Schindel et al. (2011) also found approximately $10 \%$ overestimation of a visual oddball, relative to standards.

Seifried and Ulrich (2010) recently provided evidence in a visual oddball experiment that estimates of PSE are strongly biased by the distribution of to-be-judged oddball durations, thus highlighting the context sensitivity of the observed effects. They reported that if the distribution of oddball durations is centered at a value that is less than the standard duration (i.e., in the direction of the oddball effect), then estimated PSEs will shift toward the mean oddball duration; this yields greater overestimation (i.e., a larger oddball effect) than when oddball durations are centered at a value that is equal to the standard duration. In an additional study (not reported here), we replicated the Seifried and Ulrich findings for the auditory paradigm used in the present set of experiments and also showed that shortening (rather than lengthening) is found when oddball durations are centered on a value that is longer than the standard duration. Thus, these supplementary findings extend the results of Seifried and Ulrich both to the auditory modality and to conditions that consider whether the oddball effect can be reversed (i.e., reveal underestimation) when the distribution of oddball durations is shifted to a value that is longer than the standard.

\section{Conclusions}

Two experiments have shown that for an auditory oddball paradigm, oddball pitch distance, likelihood, and position all influence the perceived duration of the deviant oddball stimulus. Oddballs that are farther in pitch from the standard and in later sequence positions are perceived to be longer than oddballs that are closer in pitch to the standard or in earlier sequence positions, with a larger difference between nearand far-pitch oddballs in low-likelihood conditions. The same pattern of duration distortions is observed regardless of whether the oddballs were higher or lower in pitch than the standard, ruling out the possibility that the pitch effect is one of absolute pitch height or attributable to differences in perceived loudness. The observed interaction between the pitch effect and oddball likelihood provides little direct support for either a repetition suppression account or an enhanced-attention account. Moreover, the positive relationship between the time required to detect an oddball stimulus and PSEs across all conditions provides some initial evidence that the oddball effect is at least partly driven by the latency to initiate timing following oddball onset. This interpretation is consistent with a temporal preparation account of the position effect. Thus, oddball stimuli that are more perceptually salient or more temporally expected are perceived to be longer in duration because they are more quickly detected.

Author Note Portions of this research were presented at the $53^{\text {rd }}$ Annual Meeting of the Psychonomic Society and at the 2012 Midwest Cognitive Science Conference. The authors gratefully acknowledge William Matthews, Virginie van Wassenhove, Åke Hellström, and the members of the Timing, Attention, and Perception (TAP) Lab at Michigan State University for their many helpful suggestions for improvements to earlier versions of the manuscript. Special thanks are also due to Karli Nave, Bradley Seegert, and Katherine Jones for their assistance with data collection and feedback throughout the research. Correspondence concerning this article should be directed to J.D. McAuley (email: dmcauley@msu.edu) or E. Kim (email: kimelis1@msu.edu), Department of Psychology, Michigan State University, East Lansing, MI 48824.

\section{References}

Church, R. M. (1984). Properties of the internal clock. Annals of the New York Academy of Sciences, 423, 566-582.

Drazin, D. H. (1961). Effects of foreperiod, foreperiod variability, and probability of stimulus occurrence on simple reaction time. Journal of Experimental Psychology, 62, 43-50.

Gibbon, J. (1977). Scalar expectancy theory and Weber's law in animal timing. Psychological Review, 84(3), 279-325.

Gibbon, J., Church, R. M., \& Meck, W. H. (1984). Scalar timing in memory. Annals of the New York Academy of Sciences, 423, 5277.

Gordon, I. E. (1967). Stimulus probability and simple reaction time. Nature, 215(5103), 895-896.

Grill-Spector, K., Henson, R., \& Martin, A. (2006). Repetition and the brain: Neural models of stimulus-specific effects. Trends in Cognitive Sciences, 10(1), 14-23.

Grondin, S., \& Rammsayer, T. (2003). Variable foreperiods and temporal discrimination. Quarterly Journal of Experimental Psychology: Human Experimental Psychology, 56(4), 731-765.

Hautus, M. J. (1995). Corrections for extreme proportions and their biasing effects on estimated values of d'. Behavior Research Methods, Instruments, \& Computers, 27, 46-51.

Jones, M. R. (1976). Time, our lost dimension: Toward a new theory of perception, attention, and memory. Psychological Review, 83(5), 323-355.

Jones, M. R., Johnston, H. M., \& Puente, J. (2006). Effects of auditory pattern structure on anticipatory and reactive attending. Cognitive Psychology, 53(1), 59-96.

Jones, M. R., Moynihan, H., MacKenzie, N., \& Puente, J. (2002). Temporal aspects of stimulus-driven attending in dynamic arrays. Psychological Science, 13(4), 313-319.

Klemmer, E. T. (1956). Time uncertainty in simple reaction time. Journal of Experimental Psychology, 51(3), 179-184.

Large, E. W., \& Jones, M. R. (1999). The dynamics of attending: How people track time-varying events. Psychological Review, 106(1), $119-159$. 
Lejeune, H. (1998). Switching or gating? The attentional challenge in cognitive models of psychological time. Behavioural Processes, 44(2), 127-145.

MacMillan, N. A., \& Creelman, C. D. (2005). Detection theory: A user's guide. New York: Cambridge University Press.

Matthews, W. J. (2011). Stimulus repetition and the perception of time: The effects of prior exposure on temporal discrimination, judgment, and production. PLoS One, 6(5), e19815. doi:10.1371/ journal.pone.0019815

McAuley, J. D., \& Jones, M. R. (2003). Modeling effects of rhythmic context on perceived duration: A comparison of interval and entrainment approaches to short-interval timing. Journal of Experimental Psychology. Human Perception and Performance, 29(6), $1102-1125$.

Meck, W. H. (1983). Selective adjustment of the speed of internal clock and memory processes. Journal of Experimental Psychology. Animal Behavior Processes, 9(2), 171-201.

Mo, S. S., \& George, E. J. (1977). Foreperiod effect on time estimation and simple reaction time. Acta Psychologica, 41(1), 47-59. doi:10.1016/0001-6918(77)90010-5

Niemi, P., \& Näätänen, R. (1981). Foreperiod and simple reaction time. Psychological Bulletin, 89(1), 133-162. doi:10.1037/0033-2909. 89.1.133

Pariyadath, V., \& Eagleman, D. M. (2007). The effect of predictability on subjective duration. PLoS One, 2(11), e1264.

Pariyadath, V., \& Eagleman, D. M. (2012). Subjective duration distortions mirror neural repetition suppression. PLoS One, 7(12), e49362. doi:10.1371/journal.pone.0049362
Penton-Voak, I. S., Edwards, H., Percival, A., \& Wearden, J. H. (1996). Speeding up an internal clock in humans? Effects of click trains on subjective duration. Journal of Experimental Psychology. Animal Behavior Processes, 22(3), 307-320.

Rose, D., \& Summers, J. (1995). Duration illusions in a train of visual stimuli. Perception, 24(10), 1177-1187.

Schindel, R., Rowlands, J., \& Arnold, D. H. (2011). The oddball effect: Perceived duration and predictive coding. Journal of Vision, 11(2).

Seifried, T., \& Ulrich, R. (2010). Does the asymmetry effect inflate the temporal expansion of odd stimuli? Psychological Research, 74(1), 90-98.

Treisman, M. (1963). Temporal discrimination and the indifference interval: Implications for a model of the "internal clock". Psychological Monographs: General and Applied, 77(13), 1-31.

Treisman, M., Faulkner, A., Naish, P. L., \& Brogan, D. (1990). The internal clock: Evidence for a temporal oscillator underlying time perception with some estimates of its characteristic frequency. Perception, 19(6), 705-743. doi:10.1068/p190705

Tse, P. U., Intriligator, J., Rivest, J., \& Cavanagh, P. (2004). Attention and the subjective expansion of time. Perception \& Psychophysics, 66(7).

Ulrich, R., Nitschke, J., \& Rammsayer, T. (2006). Perceived duration of expected and unexpected stimuli. Psychological Research, 70(2), $77-87$.

van Wassenhove, V., Buonomano, D. V., Shimojo, S., \& Shams, L. (2008). Distortions of subjective time perception within and across senses. PLoS One, 3(1), e1437. 